\title{
Acetylation of alcohols, phenols and salicylic acid by heteropoly acids in acetic anhydride: a green and eco-friendly protocol for synthesis of acetyl salicylic acid (Aspirin)
}

\author{
Majid M. Heravi,* Farahnaz K. Behbahani, and Fatemeh F. Bamoharram \\ Department of Chemistry, School of Sciences, Azzahra University, Vanak, Tehran, Iran \\ E-mail: mmh1331@yahoo.com
}

\begin{abstract}
A series of eco-friendly solid acid catalysts including; Preyssler, Wells-Dowsen and Keggin HPAs have been used as catalysts for acetylation of alcohols, phenols and salicylic acid with acetic anhydride. The performance of different forms of heteropoly acids in the presence of acetic anhydride as acetylating agent for acetylation of salicylic acid was compared. In all cases, the best conditions were observed using Preyssler as catalyst.
\end{abstract}

Keywords: Salicylic acid, alcohols, phenols, heteropoly acids, acetylation, Aspirin

\section{Introduction}

Acetylation of alcohols is an important and routinely utilized transformation in organic chemistry ${ }^{1}$. Among the various protecting groups used for the hydroxyl group, acetyl is one of the most common groups, being stable in the acid reaction conditions and also ease of removal by mild alkaline hydrolysis. The most commonly used reagent combination for this reaction uses acid anhydride in the presence of acid or base catalysts ${ }^{2}$. Various metal salts such as $\mathrm{CoCl}_{2}{ }^{3}$, $\mathrm{TiCl}_{4}-\mathrm{AgClO}_{4}{ }^{4}, \mathrm{TaCl}_{5}$ and $\mathrm{TaCl}_{5}-\mathrm{SiO}_{2}{ }^{5}, \mathrm{Ce}(\mathrm{III})$ triflate ${ }^{6}, \mathrm{Sn}(\mathrm{IV})$ porphyrine ${ }^{7}$ and some metal triflates such as $\mathrm{Sc}(\mathrm{OTf})_{3}{ }^{8} \operatorname{MeSiOTf}^{9}, \operatorname{In}(\mathrm{OTf})_{3}{ }^{10}, \mathrm{Cu}(\mathrm{OTf})_{2}{ }^{11}$ and $\mathrm{Bi}(\mathrm{OTf})_{3}{ }^{12}$ bis(cyclopentadienyl) zirconium dichloride ${ }^{13}, \mathrm{I}_{2}{ }^{14}$, 1,3-dibromo-5,5-dimethylhydentoin or trichloroisocyanuric acid ${ }^{15}$ have been investigated to meet the demand for more efficient and selective methods. In spite of these waves of interest due to the importance of acetylation, introduction of inexpensive and green catalyst for this purpose is still in much demand.

In comparison with the liquid mineral acids, solid acids could be easily separated from the reaction mixture by simple filtration with high recovery. This advantage directly leads to a decrease in of equipment cauterization and environment pollution. Among various solid acids, heteropolyacids (HPAs) have unique physical-chemical properties. Their acidity is significantly higher than those of traditional mineral acids. Furthermore, HPAs are capable of protonating and 
activating the substrate; and in some cases, HPAs are more effective than usual inorganic acid and the traditional acid catalysts. Therefore, they are widely used as homogeneous and heterogeneous acid catalysts for the synthetic reactions ${ }^{16}$. They have very strong Brønsted acidity approaching the superacid region and this acid-base property can be varied over a wide range by changing the chemical composition.

In the last two decades the broad utility of HPAs as acid and oxidation catalysis has been demonstrated in a wide variety of synthetically useful selective transformations of organic substrates $^{17-19}$. Heteropolyacids (HPAs), presently being used in several industrial processes, are important for the so-called clean technologies since many of the environmental pollution and corrosion problems of the traditional technologies are avoided ${ }^{20-22}$. Among the HPAs, Keggin family appeared rapidly as interesting candidates for strong acid-demanding reactions. Moreover, the use of supported HPAs for both increasing the active site accessibility and limiting the formation of bulky residues as much as possible has gained interest ${ }^{23}$. If one applies the principles proposed for green chemistry will see, that Preyssler catalyst will be introduced as promising candidates for green catalysts. This catalyst is green with respect to corrosiveness, safety, quantity of waste, and separability. This heteropoly acid with fourteen acidic protons is an efficient "supper acid" solid catalyst with unique hydrolytic stability $(\mathrm{pH}=0-12)^{24}$. Furthermore, Wells-Dawson type heteropolyacids possess super-acidity and a remarkable stability both in solution and in the solid state ${ }^{25}$.

In this paper we wish to report acetylation of alcohols, phenols and salicylic acid by the various of heteropoly acids in acetic anhydride as acetylating agent without solvent. Acetylation of salicylic acid gave acetyl salicylic acid or aspirin. Since, Dreser introduced the clinical use of aspirin in 1899, it has become one of the most extensively and widely used drugs. As a consequence, it has also been one of those studied in greater depth. Even after so many years, new properties of aspirin are still being discovered today. Interest in aspirin as an important drug in oncology is basically due to the development of three lines of research. The first has shown the importance of aspirin in the inhibition of carcinogenesis processes in vitro and in vivo models $^{26,27}$.

\section{Results and Discussion}

Recently we described the acetylation of alcohols and phenols ${ }^{28}$ and direct acetylation of tetrahydropyranyl ethers ${ }^{29}$ by ferric perchlorate, acetylation of THP ethers by bismuth nitrate ${ }^{30}$, acetylation of alcohols and phenols using copper (II) sulfate ${ }^{31}$ and acetylation of alcohols and phenols using $\mathrm{H}_{14}\left[\mathrm{NaP}_{5} \mathrm{~W}_{30} \mathrm{O}_{110}\right]^{32}$. We are interested in catalytic reactions ${ }^{33}$ and catalytic applicability of heteropoly acid compounds ${ }^{34-37}$. These catalysts have crown shape. As a typical, Preyssler anion structure $\left(\left[\mathrm{NaP}_{5} \mathrm{~W}_{30} \mathrm{O}_{110}\right]^{14^{-}}\right)$have been formed from five units of $\mathrm{PW}_{6} \mathrm{O}_{22}{ }^{24}$. In continuation of our investigation on application of heteropoly acids in organic synthesis, in this paper we wish to describe the direct acetylation of alcohols, phenols to corresponding acetylated 
compounds and salicylic acid to acetylsalicylic acid. The major goal described in this method is the design and development of application for the heteropoly acids in industry. In order to extend the scope of this acetylation reaction, it was carried out on a variety of substrates using a typical procedure where (0.14-0.56 mol\%) of heteropoly acids and 1-1.5 equivalent of substrate to acetic anhydride were added at room temperature. The general efficiency of this reaction is evident from the variety of hydroxyl compounds including primary, secondary, tertiary, benzylic alcohols and phenols, which react in excellent yields and relative short reaction times (Scheme 1, Table 1,2). Primary alcohols acetylated within less time than secondary and tertiary alcohols. In order to more evaluate of merit and worthy of this procedure, we tried with 1,2-pentandiol as a starting material in the presence of Wells-Dowsen heteropoly acid $\left(\mathrm{H}_{6}\left[\mathrm{P}_{2} \mathrm{~W}_{18} \mathrm{O}_{62}\right]\right)$ and acetic anhydride under the same reaction condition. After 120 minutes, we only obtained 2hydroxypentyl acetate (entry 14, Table 1). It is very interesting to note that tertiary and secondary alcohols such as t-butanol and 4-methyl-2-pentyl alcohol (entries 4 and 2, Table 1,2) can also be acetylated with satisfactory yields and there was no elimination product in the mixture.

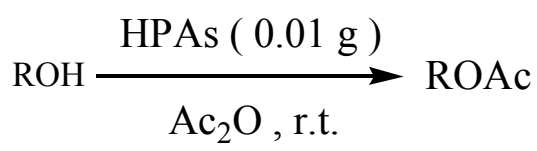

R: benzy-, allyl-, n-amyl-, propargyl-, cyclohexyl-, 2-methyl cyclohexyl-, tert-butyl-, cinnamyl, 3-methyl phenyl-, phenyl-,4-nitro phenyl-, 2-butyl-, 2-chloro benzyl-, 4-methoxy benzyl-,4methyl benzyl-, isoamyl-, 2-furyl-, 4-methyl 2-pentyl-

\section{Scheme 1}

This method tolerates other functionalities on the substrate such as double and triple bonds. It was, in fact, possible to obtain easily the quantitative acetylation of propargyl and allyl alcohols (entries, 8 and 9, Table 1,2). Withdrawing, and releasing electrons groups on moiety of phenols were converted to the corresponding phenyl acetates in excellent yields and short reaction time (entries 10, 11 and 12, Table 1,2). Comparison of Preyssler, Wells-Dowsen and Keggin HPAs, showed that the activity was higher for $\mathrm{H}_{14}\left[\mathrm{NaP}_{5} \mathrm{~W}_{29} \mathrm{MoO}_{110}\right]$. In other words, $\mathrm{H}_{14}-\mathrm{P}_{5} \mathrm{Mo}$ is a better catalyst for acetylation of alcohols and phenols by acetic anhydride. It seems clear that the acid strength of the protons on $\mathrm{H}_{14}-\mathrm{P}_{5} \mathrm{Mo}$ is sufficient to catalyze the acetylation reactions and potentially all protons are active sites. 
Table 1. Preyssler $\left\{\mathrm{H}_{14}\left[\mathrm{NaP}_{5} \mathrm{~W}_{29} \mathrm{MoO}_{110}\right]\right\}$ and Wells-Dowsen $\left\{\mathrm{H}_{6}\left[\mathrm{P}_{2} \mathrm{~W}_{18} \mathrm{O}_{62}\right]\right\}$ heteropoly acids catalyzed acetylation of alcohols and phenols in acetic anhydride

\begin{tabular}{|c|c|c|c|c|}
\hline \multirow{2}{*}{ Entry } & \multirow{2}{*}{ Substrate } & \multirow{2}{*}{ Product } & $\mathrm{H}_{14}\left[\mathrm{NaP}_{5} \mathrm{~W}_{29} \mathrm{MoO}_{110}\right]$ & $\mathrm{H}_{6}\left[\mathrm{P}_{2} \mathrm{~W}_{18} \mathrm{O}_{62}\right]$ \\
\hline & & & Time(min)/ Yield\% & Time(min)/ Yield $\%$ \\
\hline 1 & & & $15 / 94$ & $120 / 96$ \\
\hline 2 & & & $30 / 92$ & $120 / 85$ \\
\hline 3 & & & $10 / 91$ & $30 / 88$ \\
\hline 4 & & & $75 / 89$ & $180 / 87$ \\
\hline 5 & & & $5 / 98$ & $35 / 98$ \\
\hline 6 & & & $15 / 95$ & $40 / 95$ \\
\hline 7 & & & $30 / 93$ & $30 / 93$ \\
\hline 8 & & & $15 / 89$ & $90 / 89$ \\
\hline 9 & & & $30 / 91$ & $300 / 91$ \\
\hline 10 & & & $40 / 87$ & $300 / 87$ \\
\hline 11 & & & $10 / 95$ & $400 / 95$ \\
\hline 12 & & & $10 / 91$ & $400 / 91$ \\
\hline 13 & & & $15 / 89$ & $400 / 89$ \\
\hline 14 & & & --- & $120 / 85$ \\
\hline
\end{tabular}


Table 2: Keggin heteropoly acids catalyzed acetylation of alcohols and phenols in acetic anhydride

\begin{tabular}{|c|c|c|c|c|c|c|c|}
\hline \multirow[b]{2}{*}{ Entry } & \multirow[b]{2}{*}{ Substrate } & \multirow[b]{2}{*}{ Product } & $\underline{\mathrm{H}_{5}\left[\mathrm{P} \mathrm{Mo}_{10} \mathrm{~V}_{2} \mathrm{O}_{40}\right]}$ & $\underline{\mathrm{H}_{4}\left[\mathrm{PMo}_{11} \mathrm{~V}_{1} \mathrm{O}_{40}\right]}$ & $\mathrm{H}_{4}\left[\mathrm{Si} \mathrm{Mo}{ }_{12} \mathrm{O}_{40}\right]$ & $\mathrm{H}_{3}\left[\mathrm{P} \mathrm{Mo}_{12} \mathrm{O}_{40}\right]$ & $\underline{\mathrm{HNa}_{2}\left[\mathrm{PW}_{12} \mathrm{O}_{40}\right]}$ \\
\hline & & & $\begin{array}{l}\text { Time(min)/ } \\
\text { Yield\% }\end{array}$ & Time $(\min ) /$ Yield $\%$ & $\begin{array}{l}\text { Time }(\min ) / \\
\text { Yield\% }\end{array}$ & $\begin{array}{l}\text { Time }(\min ) / \\
\text { Yield\% }\end{array}$ & $\begin{array}{l}\text { Time(min)/ } \\
\text { Yield\% }\end{array}$ \\
\hline 1 & $\begin{array}{l}\text { cyclohexan } \\
\text { ol }\end{array}$ & $\begin{array}{l}\text { Cyclo hexyl } \\
\text { acetate }\end{array}$ & $30 / 95$ & $30 / 92$ & $30 / 90$ & $30 / 94$ & $30 / 90$ \\
\hline 2 & $\begin{array}{l}\text { 4-methyl-2- } \\
\text { pentanol }\end{array}$ & $\begin{array}{l}\text { 4-methyl-2- } \\
\text { pentyl } \\
\text { acetate }\end{array}$ & $30 / 90$ & $60 / 88$ & $90 / 93$ & $60 / 92$ & $90 / 91$ \\
\hline 3 & $\begin{array}{l}\text { n-amyl } \\
\text { alcohol }\end{array}$ & $\begin{array}{l}\text { n-amyl } \\
\text { acetate }\end{array}$ & $30 / 91$ & $30 / 91$ & $30 / 91$ & $30 / 93$ & $30 / 88$ \\
\hline 4 & $\begin{array}{l}\text { tert-butyl } \\
\text { alcohol }\end{array}$ & $\begin{array}{l}\text { tert-butyl } \\
\text { acetate }\end{array}$ & $90 / 92$ & $120 / 90$ & $150 / 87$ & $120 / 88$ & $180 / 91$ \\
\hline 5 & $\begin{array}{l}\text { 4-methoxy } \\
\text { benzyl } \\
\text { alcohol }\end{array}$ & $\begin{array}{l}\text { 4-methoxy } \\
\text { benzyl } \\
\text { acetate }\end{array}$ & $20 / 96$ & $15 / 97$ & $30 / 89$ & $10 / 98$ & $15 / 98$ \\
\hline 6 & $\begin{array}{l}\text { benzyl } \\
\text { alcohol }\end{array}$ & $\begin{array}{l}\text { Benzyl } \\
\text { acetate }\end{array}$ & $25 / 95$ & $20 / 95$ & $60 / 95$ & $15 / 95$ & $30 / 95$ \\
\hline 7 & $\begin{array}{l}\text { 4-methyl } \\
\text { benzyl } \\
\text { alcohol }\end{array}$ & $\begin{array}{l}\text { 4-methyl } \\
\text { benzyl } \\
\text { acetate }\end{array}$ & $30 / 93$ & $30 / 93$ & $40 / 93$ & $15 / 91$ & $15 / 93$ \\
\hline 8 & $\begin{array}{l}\text { 4-nitro } \\
\text { benzyl } \\
\text { alcohol }\end{array}$ & $\begin{array}{l}\text { 4-nitro } \\
\text { benzyl } \\
\text { acetate }\end{array}$ & $40 / 85$ & $30 / 88$ & $150 / 94$ & $20 / 86$ & $45 / 90$ \\
\hline 9 & allyl alcohol & $\begin{array}{l}\text { Allyl } \\
\text { acetate }\end{array}$ & $40 / 91$ & $90 / 91$ & $120 / 91$ & $20 / 91$ & $150 / 91$ \\
\hline 10 & $\begin{array}{l}\text { propargyl } \\
\text { alcohol }\end{array}$ & $\begin{array}{l}\text { Propargyl } \\
\text { acetate }\end{array}$ & $40 / 87$ & $75 / 87$ & $120 / 87$ & $60 / 87$ & $150 / 87$ \\
\hline 11 & phenol & $\begin{array}{l}\text { phenyl } \\
\text { acetate }\end{array}$ & $10 / 95$ & $35 / 95$ & $180 / 95$ & $35 / 95$ & $60 / 95$ \\
\hline 12 & $\begin{array}{l}\text { p-cresol } \\
\text { p-cresol }\end{array}$ & $\begin{array}{l}\text { p-cresyl } \\
\text { acetate }\end{array}$ & $10 / 91$ & $20 / 91$ & $180 / 91$ & $15 / 91$ & $30 / 91$ \\
\hline 13 & $\begin{array}{l}\text { 4-nitro } \\
\text { phenol }\end{array}$ & $\begin{array}{l}\text { 4-nitro } \\
\text { phenyl } \\
\text { acetate }\end{array}$ & $15 / 89$ & $40 / 89$ & $210 / 89$ & $40 / 89$ & $45 / 89$ \\
\hline
\end{tabular}


Ongoing we used Keggin HPAs (Table 2) for these reactions. The results of the comparison among the Keggin HPAs $\left(\mathrm{H}_{5}\left[\mathrm{PMo}_{10} \mathrm{~V}_{2} \mathrm{O}_{40}\right], \mathrm{H}_{4}\left[\mathrm{PMo}_{11} \mathrm{~V}_{1} \mathrm{O}_{40}\right], \mathrm{H}_{4}\left[\mathrm{Si} \mathrm{Mo}_{12} \mathrm{O}_{40}\right], \mathrm{H}_{3}\left[\mathrm{P} \mathrm{Mo}_{12} \mathrm{O}_{40}\right]\right.$ and $\mathrm{HNa}_{2}\left[\mathrm{PW}_{12} \mathrm{O}_{40}\right]$ showed, they are almost same regarding the yields and reaction times. However using $\mathrm{H}_{4}\left[\mathrm{Si} \mathrm{Mo}_{12} \mathrm{O}_{40}\right]$, as catalyst needed longer reaction times. In Keggin HPAs, amount of proton effect didn't observed to increase rate of reactions. We also used $\mathrm{H}_{6}\left[\mathrm{P}_{2}\right.$ $\mathrm{W}_{18} \mathrm{O}_{62}$ ] as a Wells-Dowsen HPA (Table1) for acetylation of alcohols and phenols. The results showed that the activity was lower for $\mathrm{H}_{6}\left[\mathrm{P}_{2} \mathrm{~W}_{18} \mathrm{O}_{62}\right]$. Besides these HPAs, we examined others HPAs including $\left(\mathrm{PW}_{11}, \mathrm{PW}_{11} \mathrm{O}_{39}, \mathrm{PW}_{11} \mathrm{Ni}, \mathrm{PW}_{11} \mathrm{NiO}_{40}, \mathrm{PW}_{11} \mathrm{Zn}, \mathrm{PW}_{11} \mathrm{ZnO}_{40}, \mathrm{~K}_{8}\left[\mathrm{SiW}_{11} \mathrm{O}_{39}\right]\right.$ ) for acetylation of benzyl alcohol in acetic anhydride (Table 4). These HPAs were not efficient. For the salve of application of this method and development of a green way for synthsis of acetylsalicylic acid (aspirin), we used salicylic acid as a substrate (Table 3).

Table 3: Acetylation of salicylic acid by various heteropoly acids in acetic anhydride at room temperature

\begin{tabular}{cccc}
\hline Entry & Substrate & Time $(\mathrm{h})$ & Yield\% \\
\hline 1 & $\mathrm{H}_{6}\left[\mathrm{P}_{2} \mathrm{~W}_{18} \mathrm{O}_{62}\right]$ & 0.5 & 91 \\
2 & $\mathrm{HNa}_{2}\left[\mathrm{PW}_{12} \mathrm{O}_{40}\right]$ & 1.5 & 92 \\
3 & $\mathrm{H}_{3}\left[\mathrm{P} \mathrm{Mo}_{12} \mathrm{O}_{40}\right]$ & 1.0 & 88 \\
4 & $\mathrm{H}_{4}\left[\mathrm{Si} \mathrm{Mo}_{12} \mathrm{O}_{40}\right]$ & 0.5 & 94 \\
5 & $\mathrm{H}_{4}\left[\mathrm{PMo}_{11} \mathrm{~V}_{1} \mathrm{O}_{40}\right]$ & 1.2 & 86 \\
6 & $\mathrm{H}_{5}\left[\mathrm{P} \mathrm{Mo}_{10} \mathrm{~V}_{2} \mathrm{O}_{40}\right]$ & 0.5 & 93 \\
7 & $\mathrm{H}_{14}\left[\mathrm{NaP}_{5} \mathrm{~W}_{29}\right.$ & 2.0 & 91 \\
8 & $\left.\mathrm{MoO}_{110}\right]$ & 2.0 & 87 \\
\hline
\end{tabular}

Table 4. Comparison of among some of HPAs in acetylation of benzyl alcohol with acetic anhydride at room temperature

\begin{tabular}{cccc}
\hline Entry & $\mathrm{HPAs}$ & Time $(\mathrm{h})$ & Yield $\%$ \\
\hline 1 & $\mathrm{PW}_{11}$ & 30 & 40 \\
2 & $\mathrm{PW}_{11} \mathrm{O}_{39}$ & “ & 40 \\
3 & $\mathrm{PW}_{11} \mathrm{Ni}$, & “ & 30 \\
4 & $\mathrm{PW}_{11} \mathrm{NiO}_{40}$ & “ & 30 \\
5 & $\mathrm{PW}_{11} \mathrm{Zn}$ & “ & 45 \\
6 & $\mathrm{PW}_{11} \mathrm{ZnO}_{40}$ & “ & 45 \\
7 & $\mathrm{~K}_{8}\left[\mathrm{SiW}_{11} \mathrm{O}_{39}\right]$ & 24 & 80 \\
\hline
\end{tabular}




\section{Experimental Section}

\section{Acetylation of alcohols and phenols catalyzed by HPAs. Typical procedure}

Benzyl alcohol (1 mmol, $0.12 \mathrm{~g})$, acetic anhydride $(1.5 \mathrm{mmol}, 0.18 \mathrm{ml})$ and $\operatorname{HPAs}(0.01 \mathrm{~g})$ were mixed. The mixture was stirred at room temperature at indicated time (Table 1-3). Upon completion of the reaction monitored by (TLC) the reaction mixture was diluted with sodium bicarbonate $10 \%(15 \mathrm{ml})$ and extracted with $\mathrm{CH}_{2} \mathrm{Cl}_{2}(3 \times 20 \mathrm{~mL})$. Combined organic layers were dried over $\mathrm{Na}_{2} \mathrm{SO}_{4}$, the solvent was removed by evaporation to afford the acetylated compound.

\section{Acetylation of salicylic acid catalyzed by HPAs}

Salicylic acid (1 mmol, $0.138 \mathrm{~g})$, acetic anhydride $(1.5 \mathrm{mmol}, 0.18 \mathrm{~mL})$ and HPAs $(0.01 \mathrm{~g})$ were mixed. The mixture was stirred at room temperature at idicated time (Table 4). Upon completion of the reaction monitored by (TLC) the reaction mixture was diluted with water and mixed for 5 minutes. The product was filtered, washed with water $(10 \mathrm{~mL})$ and dried in $90^{\circ} \mathrm{C}$. At the end of work up, mother liquid evaporated to dryness to the afford catalyst. We repeated this reaction with $10 \mathrm{mmol}$ of salicylic acid and obtained results as well as those of with $1 \mathrm{mmol}$ of salicylic acid. Then, this procedure could be used in large scale even more than $10 \mathrm{mmol}$ of starting material.

\section{Conclusions}

Inexpensive, eco-friendly, recyclable and available heteropolyacids is an efficient solid acid catalyst for acetylation of alcohols, phenols and salicylic acid in acetic anhydride were used. One of important feature of these solid acids is high thermal and hydrolytic stability throughout a wide $\mathrm{pH}$ range. Thus, wide range of catalytic reactions can be affected without loss of structure and activity. The results also suggest that at the same conditions including: the molar ratio, reaction time, temperature and alcohol type; Preyssler catalyst is most efficient HPAs for this purpose. Finally, simple experimental set up and procedure makes this method a useful addition to the methodologies that require solid catalysts with strong acidic strength, highly thermal stability and functionality over a wide range of $\mathrm{pH}$, especially for synthesis of aspirin.

\section{References}

1. (a) Greene, T.W.; Wuts, P. G. M.; Protection Groups in Organic Synthesis 3rd Edn. John Wiley: New York, 1999. (b)Sartori, G.; Ballini, R.; Bigi, F.; Bosica, G.; Maggi, R.; Righi, P. Chem. Rev. 2004, 104, 199.

2. Pearson, A. L.; Roush, W. J.; Handbook of Reagents for Organic Synthesis: Acetylating Agents and Protecting Groups, John Wiley, Chichester, 1999. 
3. (a) Harton, D. Organic Synthesis Collective Vol. V, 1991, Wiley: New York. (b) Zhdarov, R. L.; Zherodarova, S. M.; Synthesis 1975, 222. (c) Steglich, W.; Hoefle, G. Angew. Chem. Int. Ed. 1969, 8, 981. (d) Hoefle, G.; Steglich, W.; Vorbrueggen, H. Angew. Chem. Int. Ed. 1978, 17, 569. (e) Scriven, E. F. V. Chem. Soc. Rev. 1983, 12, 129. (f) Sano, T.; Ohaschi, K.; Oreyama, T. Synthesis 1999, 1141. (g) Vedejs, E.; Bennett, N. S.; Conn, L. M. L.; Diver, S. T.; Gingras, M.; Lin, S.; Oliver, P. A.; Peterson, A. L. J. Org. Chem. 1993, 58, 7258. (h) Vedejs, E.; Diver, S. T.; J. Am. Chem. Soc. 1993, 115, 3358. (i) Vedejs, E.; Daugulis, O.; Diver, S. T. J. Org. Chem. 1996, 61, 430.

4. Cope, A. C.; Herrich, E. C. Organic Synthesis Collective, Vol. IV, Wiley: New York, 1963.

5. Chandrasekhar, S.; Chander, T. R.; Takhi, M. Tetrahedron Lett. 1998, 39, 3263.

6. Dalpozzo, R.; De Nino, A.; Maiuolo, L.; Procopiou, P. A.; Nardi, M.; Bartoli, G.; Romeo, R.; Tetrahedron Lett. 2003, 44, 5621.

7. Moghadam, M.; Tangestaninejad, S.; Mirkhani, V.; MohammadpourBaltrork, I.; Shabani, R.; J. Mol. Catal. 2004, 219, 73.

8. Ishihara, K.; Kubota, M.; Kurihara, H.; Yamamoto, H.; J. Org. Chem. 1996, 61, 4560.

9. Procopiou, P. A.; Baugh, S. P. D.; Flack, S. S, Inglis, G. G. A. J. Org. Chem. 1998, 63, 2342.

10. Chauhan, K. K.; Frost, C.G.; Love, I.; Waite, D. Synlett 1999, 1743.

11. Chandra, D.; Saravanan, P.; Singh, R. K.; Singh, V. K. Tetrahedron 2002, 58, 1369.

12. 12. Orita, A.; Tañáis, G.; Kakuda, A.; J. Angew. Chem. Int. Ed., 2000, 39, 2877.

13. Kantam, M. L.; Aziz, K.; Likhar, P. R. Catal. Commun. 2006, 7, 484.

14. Bosco, J. W. J.; Agrahari, A.; Saikia, A. K. Tetrahedron Lett. 2006, 47, 4065.

15. Zolfigol, M. A.; Khazaei, A.; Choghamarani, A.G.; Rostami, A.; Hajjami, M. Catal. Commun. 2006, 7, 399.

16. Timofeeva, M. M. Appl. Catal. A Gen. 2003, 256, 19.

17. a) Ono, Y.; Thomas, J. M.; Zamaraev, K. I., Eds.; Perspective in Catalysis, Blackwell: London, 1992. (b) Kozhevnikov, I. V. Catalysis by Polyoxometalates, Wiley: Chichester, 2002. (c) Mizuno, N.; Misono, M. Chem. Rev. 1998, 98, 199. (d) Izumi, Y.; Urabe, K.; Onaka, M.; Zeolite, Clay and Heteropoly Acid in Organic Reactions, Kodansha/VCH: Tokyo, 1990. (e) Hill, C. L.; Prosser-McCartha, C. M. Coord. Chem. Rev. 1995, 143, 407.

18. (a) Misono, M. Catal. Rev. Sci. Eng., 1987, 29, 269. (b) Misono, M. Catal. Rev. Sci. Eng. 1988, 30, 339 .

19. Firouzabadi, H.; Jafari, A. A. J. Iran. Chem. Soc. 2005, 2, 85.

20. Misono, M.; Nojiri, N. Appl. Catal. 1990, 64, 1.

21. Kozhevnikov, V. Chem. Rev. 1998, 98,171.

22. Mizuno, N.; Misono, M. Chem. Rev. 1998, 98, 199.

23. Rafiee, E.; Shahbazi, F. J. Mol. Catal. A: Chemical 2006, $250,57$.

24. Alizadeh, M. H.; Harmalker, S. P.; Jeannin, Y.; Martin-Frere, J.; Pope, M. T.; J. Am. Chem. Soc. 1985, 107, 2662.

25. Briand, L. E.; Baronetti, G. T.; Thomas, H. J. Appl. Catal. A: Gen. 2003, 256, 37. 
26. Seed, M.; Brown, J. R.; Freemantle, C. Cancer Res. 1997, 57, 1625.

27. Masahiko, T.; Kawano, S.; Tsuji, S.; Sawaoka, H.; Hori, M.; Du Bois, R. N. Cell 1998, 93, 705.

28. Heravi, M. M.; Behbahani, F. K.; Hekmat Shoar, R.; Oskooie, H. A. Catal. Commun. 2006, 7, 136.

29. Heravi, M. M.; Behbahani, F. K.; Hekmat Shoar, R.; Oskooie, H. A. J. Mol. Catal. A: Chemical 2006, 244, 8.

30. Asadolah, K.; Heravi, M. M. Phosphorus, Sulfur Silicon 2004, 179, 2335.

31. Heravi, M. M.; Behbahani, F. K.; Zadsirjan, V.; Oskooie, H. A. J. Braz. Chem. Soc. 2006, $17,1045$.

32. Heravi, M. M.; Behbahani, F. K.; Bamoharram, F. F. J. Mol. Catal. A: Chemical 2006, 253, 16.

33. (a) Bamoharram, F. F.; Heravi, M. M.; Roshani, M.; Gharib, A.; Jahangir, M. Appl. Catal. 2006, 302, 42. (b) Heravi, M. M.; Hekmatshoar, R.; Pedram, L.; J. Mol. Catal. A: Chem. 2005, 231, 89. (c) Heravi, M. M.; Bakhtiari, K.; Bamoharram, F. F.; Catal. Commun. 2006, 7, 373. (d) Bamoharram, F. F.; Heravi, M. M.; Roshani, M.; Gharib, A.; Jahangir, M. J. Mol. Catal. A: Chem. 2006, 252, 90. (e) Tajbakhsh, M.; Heravi, M. M.; Mohajerani, B.; Ahmadi, A. N. J. Mol. Catal. A: Chem. 2005, 236, 213.

34. Alizadeh, M. H.; Razavi, H.; Bamoharram, F. F.; Daneshvar, K. J. Mol. Catal. 2003, 206, 89.

35. Alizadeh, M. H.; Razavi, H.; Bamoharram, F. F.; Hassanzadeh, M. H. Kinet. Catal. 2003, 44, 524.

36. Bamoharram, F. F.; Heravi, M. M.; Roshani, M.; Gahangir, M.; Gharib, A. Appl. Catal. 2006, 302, 42 .

37. (a) Bamoharram, F. F.; Heravi, M. M.; Roshani, M.; Tavakoli, N. J. Mol. Catal. 2006, 252, 219. (b) Heravi, M. M.; Motamedi, R.; Seifi, N.; Bamoharram, F. F. J. Mol. Catal. 2006, 249, 1. (c) Bamoharram, F. F.; Roshani, M.; Alizadeh, M. H.; Razavi, H.; Moghayadi, M. J. Braz. Chem. Soc. 2006, 17, 505. (d) Bamoharram, F. F.; Heravi, M. M.; Roshani, M.; Akbarpour, M. J. Mol. Catal. 2006, 255,193. 